\title{
Assessment of Oral Cancer Awareness among Dental Students, Practitioners and Patients in Manitoba, Canada
}

\author{
Reda Elgazzar* Adnan Shah and Israa Elgazzar \\ College of Dentistry, University of Manitoba, Dental Diagnostic \& Surgical Sciences, Canada
}

Submission: August 17, 2018; Published: August 29, 2018

*Corresponding author: Reda Elgazzar, University of Manitoba, Dental Diagnostic \& Surgical Sciences, Canada, Tel: +1 (204) 789-3634;

Fax: +1 (204) 272-3077; Email: reda.elgazzar@umanitoba.ca

Abstract

Objectives: This study aimed at assessing the level of knowledge and awareness regarding oral cancer (OC) \& its associated manifestations and risk factors among dental students, dental practitioners, \& dental patients at the University of Manitoba, Canada. The results of this study may aid us to recognize whether a change is required to enhance our curriculum, focus our continuous education (CE) and raise the public's dental health awareness.

Materials and Methods: This study involved preparation of three different focused questionnaires relevant to each of the following categories: 1) The final year dental students at the University of Manitoba (33 students); 2) The dental practitioners working in the City of Winnipeg, Manitoba (51 of 100 returned the survey); 3) The patients attending the outpatient OMS clinic at the University of Manitoba (53 patients).

Results: About $55 \%$ of the students thought that they had received sufficient didactic \& clinical information regarding oral cancer during their U/G study, $18 \%$ thought they had inadequate information, $24 \%$ were not sure. Only $37 \%$ of the GDP thought they had sufficient knowledge concerning the prevention \& detection of oral cancer and $29 \%$ thought they had inadequate information, $33 \%$ were not sure. Fifty nine percent of the GDP had shared in the diagnosis of 1-4 Oral Cancer lesions in the last 5 years. Ninety-two percent were in favor of having more CE on oral cancer topics. Ninety-six percent of the surveyed patients knew that tobacco use might cause oral cancer; surprisingly, only $43 \%$ knew that Alcohol is a risk factor for oral cancer. Only $19 \%$ of the patients reported that they had enough information about oral cancer and that the dentist was the main source of information.

Conclusion: This study indicated that a significant percentage of the surveyed dental students and dental practitioners might need more didactic/ clinical information \& CE regarding oral cancer. Innovative programs is recommended for raising oral cancer awareness among the public and the dental patients particularly among those who are at risk of oral cancer.

Keywords: Oral cancer awareness; Dental professionals; Dental Students and Dental patients

\section{Introduction}

The World Health Organization and the International Agency for Research on Cancer (2003) acknowledged oral carcinoma as the most common neoplasm of the head and neck, with nearly 390,000 new cases per year. Worldwide, the problem is even greater with about 640,000 new cases each year. It was reported that the mortality for oral cancer is higher than in cancers such as cervical cancer, Hodgkin's lymphoma, laryngeal cancer, testicular cancer, and endocrine system cancers. This is attributed to the late stage of diagnosis and not due to the aggressiveness of the oral cancer. Another obstacle to early diagnosis is that some malignant oropharyngeal lesions, particularly the HPV16 positive ones, do not produce any visible signs, or discolorations that are usually the early warning signs of oral cancer [1-3]. Discovery of oral cancer often occurs when the cancer has metastasized to a different location, usually the lymph nodes of the neck. Long-term prognosis at this stage of diagnosis is significantly worse than if it was diagnosed in a localized intra oral area. At these more advanced stages, the primary tumor has had time to invade deep into local tissues (Figures $1 \mathrm{a} \& 1 \mathrm{~b}$ ). Oral cancer is particularly dangerous because it has a high risk of producing secondary tumors and even tertiary ones. Patients rarely have symptoms at this point. Patients who survive the first bout with oral cancer have a 20 times chance of recurrence. The heightened risk factor can last for 5-10 years after the first occurrence [3]. All the above raises the warning flags of how deadly this disease could be. At every dental visit, a complete head and neck exam should be completed manually by the dentist. 


\section{Global Journal of Otolaryngology}

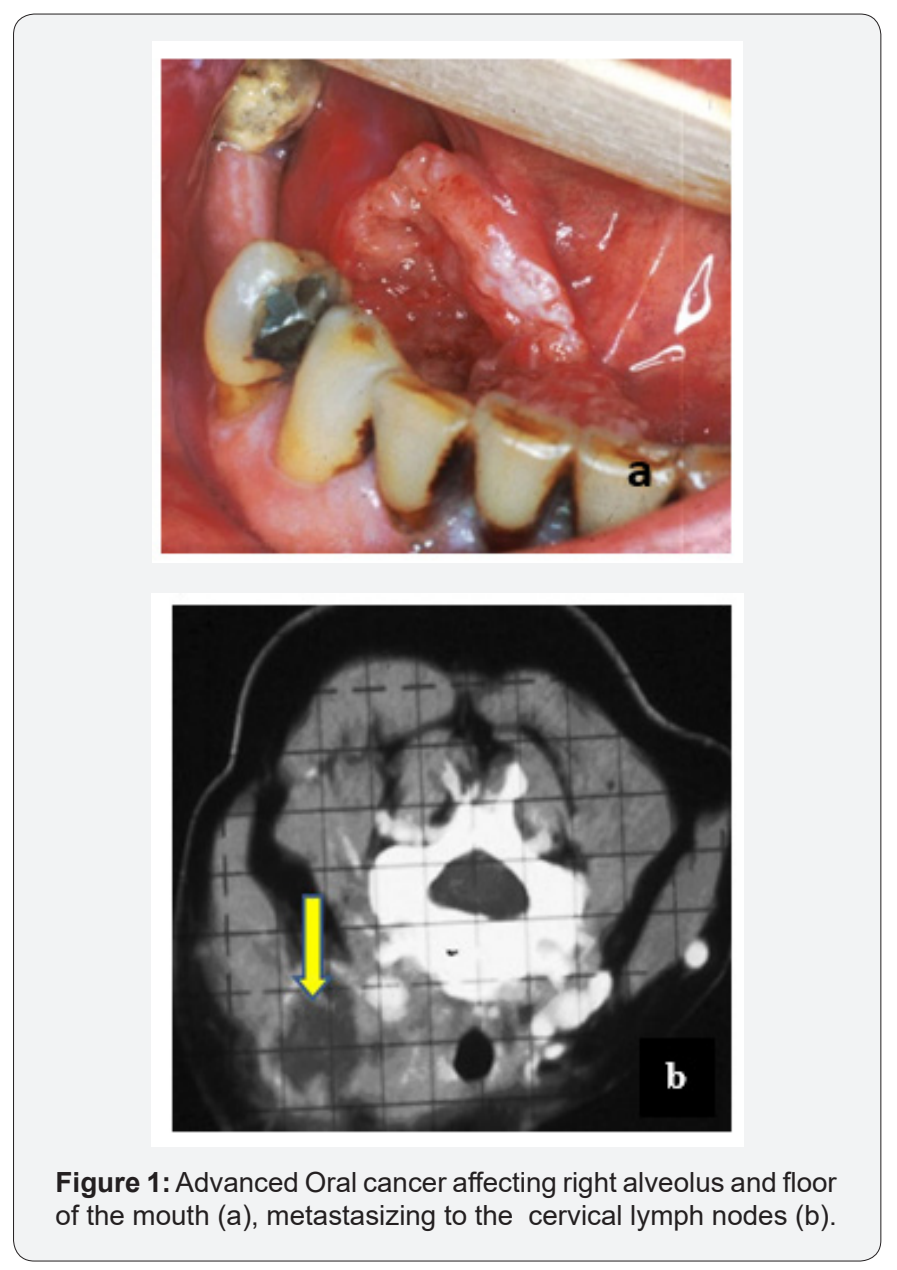

The fact that oral cancers are preventable diseases present an irony on the high incidence rates around the world. The reason of this high incidence in comparison to the simplicity of measures that can reduce the morbidity \& mortality of such diseases lies in the lack of awareness among all sectors of the community including medical and dental professionals [4]. Recently, with the current continuous change in people's preferences to engage in reading activities turning with full capacity to audio \& visual means of media reduced the effectiveness of printed brochures, leaflets, \& awareness cards in changing \& prioritizing people's attitude towards seeking proper oral care \& preventive methods for oral cancers. Awareness among academics \& professionals is also decreasing due to the previously mentioned reason \& due to the fast pace of urban life. Multimedia, videos, podcasts, wikis, \& social network programs such as "Facebook", are all now available on computers \& mobiles, \& they can collectively play a massive role in increasing awareness with regards to the importance of early detection \& prevention of cancer [5]. Successful Healthcare disease prevention programs are the fruit of awareness activities, \& their financial \& developmental impact on communities \& countries is very well established. Good Planning of effective oral cancer awareness activities is the fruit of comprehensive assessment \& surveys of the knowledge $\&$ attitude of all sectors of the community [5].

\section{Objectives}

Dental professions play an integral role in the detection, diagnosis and referral of oral cancer patients. It is unclear whether our dental graduates are well equipped with enough information to carry on such a crucial role. In addition, we are unsure whether our curricula have sufficient information and materials concerning Oral Cancer care. Therefore, the objectives of this study are as follows:

1. Assessment of the level of knowledge about oral cancer \& its associated factors among final year dental students in the College of Dentistry, University of Manitoba and among dental professional in Manitoba.

2. Estimation of the level of community awareness of oral cancer \& its associated factors in Winnipeg by surveying a sample of the regular dental patients.

\section{Materials and Methods}

The present study involves conducting 3 focused questionnaires to assess the oral cancer (OC) knowledge including risk factors and clinical symptoms, among a sample of 3 different populations: Final year undergraduate dental students, general dental practitioners \& patients attending dental clinics in the College of Dentistry at the University of Manitoba [6]. Self-administered questionnaires were posted to 100 dental practitioners in Winnipeg, emailed to 35 dental students who were in their final year, and handed directly to 100 dental patients. In addiᄀtion, a cover letter assured the participants anonymity and confidentiality of all the responses. The completed questionnaires were collected back from the dental practitioners via a prepaid envelop, from dental students by emails and directly collected from the dental patients. Questionnaires prepared for Dental practitioners, students and patients consisted of $12,13 \& 7$ different questions, respectfully. Mixed format including multiple-choice questions, Yes/No and fill in blanks was employed. The valid returned questionnaires included 51 from GDPs, 33 from the final year dental students and 53 from dental patients. The results of each survey category were analyzed by Minitab statistical package no 18.

\section{Results}

\section{Dental professionals Survey}

One hundred GDPs received the surveys, 51\% returned valid surveys. The average of participants' experience was 22.6 years (1-49 years), 95\% of them were private GDPs. $92 \%$ routinely examined their patients for oral mucosa during dental appointments; however, only $66.6 \%$ always performed oral cancer screenings, $11 \%$ sometimes did and $13 \%$ had never done a screening. Ninety one percent answered yes for how to identify risk groups and risk factors for oral cancer (this sentence is confusing). Only $22 \%$ had diagnosed one oral cancer case in the past year and $8 \%$ had diagnosed 2 cases in the past year while the remaining $70 \%$ did not see any oral cancer cases in 
the past year. The number of nonmalignant lesions diagnosed by the GDP ranged from 1-150 lesions during the past year and the average for each GDP was 35 lesions [7]. With respect to clinical and radiological information, $24 \%$ believe that they are well informed, $65 \%$ somewhat informed and only $6 \%$ were not sure and $4 \%$ did not answer these questions. Fifty nine percent attended at least one educational activity about oral cancer within last 5 years. Only 38\% believe that they have sufficient knowledge concerning the prevention and detection of oral cancer.

One hundred percent were able to mention at least 2 clinical and 2 radiological manifestations of oral cancer. One hundred percent were able to identify smoking, alcohol, viruses and environmental factors as main etiological factors of oral cancer. Thiry eight percent believed that oral cancer is preventable, $30 \%$ not preventable and $32 \%$ were not sure. The majority (85\%) of the GDPs believe that patient education about oral cancer is important to very important. In case of suspicious lesions, 85\% of the GDPs will refer their patients to an Oral and Maxillofacial surgeon, $10 \%$ to an oral pathologist, $6 \%$ to an oncologist and $4 \%$ to a senior dentist. With respect to future continuous education (CE) workshops about oral cancer, 93\% said yes to CE and majority had chosen various CE formats including workshops, online webpages, flyers and TV ads. The GDPs see that the most effective way to educate the public is directly through their dentists, printed materials, TV ads, school programs.

\section{Students Survey}

All students knew the definition of oral cancer, 78.7\% knew what high-risk groups and risk factors as related to oral cancer mean. Eighty eight percent of the students considered smoking as the main etiological factor for oral cancer as compared to only $12 \%$ for alcohol consumption. Eighty eight percent were able to identify old age, male gender and smokers as main risk groups, $12 \%$ mentioned others e.g. genetics and UV exposure. $87.8 \%$ reported that they always examine the patients' oral mucosa and $12 \%$ sometimes examined the oral mucosa. Furthermore, patient screening for oral cancer was always done by $55 \%$ of students, $33 \%$ done sometimes, and $6 \%$ never done. Sixty percent answered that they had shared in the diagnosis and referral of at least one case during their school life. Only 18\% of students considered themselves well informed about oral cancer, $46 \%$ were unsure. Twenty sevent percent believed that they had sufficient didactic and clinical training about oral cancer, $67 \%$ insufficient clinical training, and $3 \%$ got poor information. Seventeen percent considered Oral cancer preventable, 18\% is not preventable \& $30 \%$ were not sure. Fifty five percent had sufficient information on prevention and early detection of oral cancer, 15\% not, and 30\% were unsure. All students were able to mention at least 2 important manifestations of oral cancer including changes in color and/or growth, ulceration, or lymphadenopathy. Finally, 94\% of students were willing to receive more information about oral cancer.

\section{Patients' survey}

Ninety eight percent of the patients answered that they care about their oral hygiene; only 55\% visited their dentists regularly. Sixty eight percent of the patients claimed that they are literate about oral disease, $26 \%$ were not sure and $6 \%$ had no idea. Only $19 \%$ have had information about oral cancer, $77 \%$ has no idea and $4 \%$ were not sure. Dentists (57\%) \& Media (28\%) \& physicians (18\%) were the main source of information for the patients. Only $43 \%$ of the patients read health care brochures. With regards to the relationship between Oral cancer and smoking, alcohol drinking, unhealthy nutrition and environmental factors, $92 \%, 43 \%, 79 \%$ and $66 \%$ of the patients reported a positive relation respectfully.

\section{Discussion}

Carcinogens, including tobacco use, alcohol drinking and viral infection, are the major risk factors of oral cancer and the only way to prevent and early detect this disease is via raising the bar of professional involvement and public awareness. The results of this study shed light on the overall knowledge and awareness among dentists, dental students and dental patients regarding the early detection of oral cancer and its associated manifestations and risk factors in Manitoba. In the current study, $78.7 \%$ of our 4 th year dental students were able to identify the high-risk groups and risk factors as related to oral cancer. Eighty eight percent of the students considered smoking as the main etiological factor for oral cancer as compared to only $12 \%$ for alcohol drinking [8]. Keser and Pekiner (2018) assessed oral cancer awareness among dental students. Their study included 99 (50\%) third-grade and 99 (50\%) fifth-grade students. The largest number of the third-and last-grade students identified tobacco $(98 \%)$ and alcohol usage $(87.4 \%)$, prior oral cancer lesions (94.9\%), viral infections (91.9\%), UV exposure (94.4\%), betel quid chewing (84.8\%), older age (62.1\%), and low consumption of fruit and vegetables (85.4\%) [8].

In our study, $100 \%$ of participating dentists were able to identify smoking, alcohol, viruses and environmental factors as main etiological factors of oral cancer. Kebabcioglu and Pekiner (2017) [9] published their study regarding oral cancer awareness among dentists who attended 101st FDI World Dental Congress, İstanbul, Turkey. Among 170 participant dentists, there were $69(40.6 \%)$ male dentists and101(59.4\%) female dentists. Largest number of them identified tobacco (98.8\%) and alcohol usage $(91.2 \%)$, prior oral cancer lesions (95.3\%), viral infections (90.0\%), UV exposure (86.5\%), and betel quid chewing (80.6\%), and lower numbers reported older age $(56.5 \%)$ and low consumption of fruit and vegetables (52.4\%) $[9,10]$. Awojobi et al. (2015) published their article on "Why don't dentists talk to patients about oral cancer", they concluded that Dentists recognized the importance of raising awareness but identified several barriers to discussions including system factors, for instance, time constraints and a lack of financial incentive, patient factors (for example, fear of invoking undue 
anxiety) and dentist factors (for example, a lack of sufficient knowledge, training and self-confidence) [10].

Up to half of oral cancer patients are diagnosed with advanced lesions. One way to try to ensure early diagnosis may involve dental professionals raising awareness of oral cancer among their patients. In this study, only $19 \%$ of our dental patients had had information regarding oral cancer, $77 \%$ has no idea and 4\% were not sure. Dentists (57\%), Media (28\%) \& physicians (18\%) were the main source of information for them. In a similar study [11]. Babiker et al. 2017 assessed the oral cancer awareness among dental patients in Omdurman, Sudan: a cross-sectional study. They reported that 57.7\% (286) of the individuals demonstrated good knowledge of signs and symptoms, whereas 49\% (139) expressed good knowledge of risk factors of oral cancer. $66.1 \%$ of the participants reported that the most common source of information about oral cancer was from the media, while $33.9 \%$ individuals, obtained knowledge from direct contact of health workers. Knowledge of risk factors associated significantly with those reported positive attitude towards oral cancer screening and those reported direct contact with health workers as a source of information. [11] Shempi et al., (2018) [12] studied patient awareness/knowledge towards oral cancer using a cross-sectional survey. They reported that $94 \%$ \& $40 \%$ respectively of their patients was found to be aware of the relation between smoking tobacco and alcohol consumption and the risk of oral cancer [12].

\section{Conclusion}

This study indicated that only $19 \%$ of our patients had received information regarding oral cancer, and that the majority of GDP and final year dental students are not well informed about early detection and diagnosis of the disease. This survey identified an existing gap in the knowledge and practices, pertaining to oral cancer, among GDP, dental students and the public, and it emphasized the need for modifying our dental curriculum to place greater emphasis on oral cancer prevention and early detection. Planning more targeted workshops on oral cancer diagnosis and prevention, and finally creation of innovative programs for raising oral cancer awareness among the public, particularly dental patients.

\section{References}

1. Stewart BW, Kleihues P (eds.) (2003) World cancer report. Oxford: OUP, World Health Organization. International Agency for Research on Cancer: Oxford University Press, USA.

2. Franceschi S, Bidoli S, Herrero R, Muñoz N (2000) Comparison of cancers of the oral cavity and pharynx worldwide: etiological clues. Oral Oncol 36(1): 106-115.

3. SCIUBBA JJ (2001) Oral cancer and its detection History-taking and the diagnostic phase of management, JADA 132(1): 12-18.

4. Boring CC, Squires TS, Tong T, Montgomery S (1994) Cancer statistics (1994) CA Cancer J Clin 44(1): 7-26.

5. Baden E (1987) Prevention of cancer of the oral cavity and pharynx. Cancer 37(1):49-62.

6. Silverman S Jr, Shillitoe EJ (1990) Etiology and predisposing factors. In: Silverman S Jr, (edn). Oral cancer. ( $3^{\text {rd }}$ edn). Atlanta, Ga: American Cancer Society p.ss 7-30.

7. National Cancer Institute (1989) Cancer statistics review 19731987. Washington, DC: US Department of Health and Human Services, National Institutes of Health, National Cancer Institute, NIH publication no. (NIH) 88-2789.

8. Keser G, Pekiner F (2018) Assessing oral cancer awareness among dental students. J Cancer Educ.

9. Kebabcioglu 0, Pekiner F (2017) Assessing oral cancer awareness among dentists. J Cancer Educ 1: 1199-1122.

10. Awojobi O, Newton J, Scott S (2015) Why don't dentists talk to patients about oral cancer? Br Dent J 218(9): 537-541.

11. Babiker T, Osman K, Mohamed S, Mohamed M, Almahdi H (2017) Oral Cancer Awareness Among Dental Patients in Omdurman, Sudan: a cross-sectional Study. BMC Oral health 17(1): 69.

12. Shimpi N, Jethwani M, Bharatkumar A, Chyou P, Glurich I, et al. (2018) Patient awareness/knowledge towards oral cancer: a cross-sectional survey. BMC Oral Health 18(1): 86.

\section{Your next submission with Juniper Publishers will reach you the below assets}

- Quality Editorial service

- Swift Peer Review

- Reprints availability

- E-prints Service

- Manuscript Podcast for convenient understanding

- Global attainment for your research

- Manuscript accessibility in different formats

( Pdf, E-pub, Full Text, Audio)

- Unceasing customer service

Track the below URL for one-step submission https://juniperpublishers.com/online-submission.php 